\title{
Photocathode materials able to sustain high currents
}

\section{Zhaozhu Li ${ }^{1}$}

Physics Department, College of William and Mary

P.O.Box 8795, Williamsburg, 23187-8795, USA

E-mail: zli@email.wm.edu

\section{Kaida Yang}

Applied Science Department, College of William and Mary

P.O.Box 8795, Williamsburg, 23187-8795, USA

E-mail: kyangeemail.wm.edu

\section{Jose Riso}

Applied Science Department, College of William and Mary P.O.Box 8795, Williamsburg, 23187-8795, USA

E-mail: jmriso@wm.edu

\section{Rosa Alejandra Lukaszew}

Physics Department, College of William and Mary

P.O.Box 8795, Williamsburg, 23187-8795, USA

E-mail: ralukaszew@wm. edu

\begin{abstract}
We will present preliminary work on photocathode materials able to sustain high currents, pertinent to the technology of accelerators and associated systems and essential to develop strategies and technologies for next generation nuclear physics accelerator capabilities. To this end, metallic photocathodes offer several clear advantages over semiconductor photocathodes because they are robust against degradation due to surface contamination and against damage resulting from conditioning or heating and can withstand high electric surface fields present at the cathode in RF accelerators. Other advantages include their very short response time (less than picoseconds) and their very long lifetime (years or longer), which is much longer than of other types of photocathodes (hours to months). However, the main problem with metallic photocathodes is the rather low quantum efficiency $(\mathrm{QE})$, even for UV radiation. A possibility to improve the QE of metallic photocathodes is to exploit surface Plasmon resonance using adequate geometries for the intended application, as well as possible cap layers able to lower the metal work function. In this way, metal photocathodes designed to support surface Plasmons could produce high electron yields by enhancing their QE.
\end{abstract}

$X V t h$ International Workshop on Polarized Sources, Targets, and Polarimetry

September 9-13, 2013

Charlottesville, Virginia, USA

1

\section{Speaker}




\section{Introduction}

Existing photocathode technology may not meet the various requirements for long photocathode lifetime, high current and repetition rate, high polarization and/or low emittance that are required for next generation light sources and nuclear physics accelerator capabilities, particularly for electron ion colliders (EIC). Specifically, next-generation light sources will need $\mathrm{MHz}$ repetition rates, and the only way to achieve that kind of performance is with an electron gun that can deliver short electron bunches with high charge, high energy, low emittance, and a very high repetition rate; new EIC proposals stipulate $50 \mathrm{~mA}$ polarized electron beam and also expect to cool nuclear particle beams using unpolarized electron beams with $~ 100 \mathrm{~mA}$ average current. Thus, we have investigated metallic photocathodes, which offer several advantages over current photocathodes for these stringent requirements but also exhibit low QE. The only way to increase the QE without adversely affecting the emissivity, is to decrease the optical penetration depth and reduce the metal reflectivity. Coupling to the suface Plasmon polariton (SPP) modes on the metal surface is the ideal solution for this problem. Thus, we have explored metallic photocathode performance by enabling Surface Plasmon Polariton excitation as well as the use of adequate over-layers to enhance performance, since it has been shown that the addition of suitable cap layers can also reduce the work function. [1]

For metal films thicker than the optical skin depth-or for bulk metal-the probability that a photon is absorbed and transported to the surface without scattering, $\eta_{t r}$, can be expressed as [2]:

$$
\eta_{t r}=\frac{1-R(\omega)}{1+\frac{\lambda_{o p t}(\omega)}{\lambda_{e-e}(\omega)}} .
$$

where $\mathrm{R}$ is the reflectivity, $\lambda$ opt is metal's optical skin depth, and $\lambda \mathrm{e}-\mathrm{e}$ is the electron mean free path. It is worth noting that it is possible to tailor a thin film system where light couples to the SPP modes on the surface yielding a perfect absorber at a chosen wavelength, hence reducing $\mathrm{R}(\omega)$ to zero. It also turns out that SPPs have shorter evanescent fields than the optical skin depth offering an additional aspect for enhanced performance using these materials. In fact strong QE increase was demonstrated using this approach by Callcott et al. [3], where a prism was coated with a thin aluminum film and the electrons were emitted from the backside.

Due to the bound nature of the SPPs, their dispersion relationship indicates that their momentum is larger than that of the incident light but there are ways to provide adequate coupling between the incident light and SPPs to overcome this. It is possible to use a prism coupler to enhance the momentum of the incident light [3,4], or to use a periodic corrugation in the metal's surface [5]. In the latter case, it has been reported that the grating parameters such as period, shape, as well as film thickness and size can be tuned for excitation of SPPs at a particular frequency and that the tuned grating would lead to an electric field intensity 
enhancement greater than 100x near the surface due to SPP excitation. [6]. In the case of a periodic grating the coupling occurs for $\mathrm{k} 0+\mathrm{m}(2 \pi / \Lambda)=\mathrm{k}_{\mathrm{SP}}$, where $\mathrm{m}$ is an integer and $\Lambda$ is the periodicity of the nanopatterned grooves, while $\mathrm{k} 0$ and $\mathrm{k}_{\mathrm{SP}}$ are the wave vectors for the incident light and the SPP respectively.

\subsection{Experiments and simulations}

We have used gratings to excite SPPs in silver thin films for the studies reported here. We have simulated the required film thickness to achieve SPPs excitation at 45 degrees for a specific wavelength $(633 \mathrm{~nm})$ using standard gratings (pitch $1.6-/+0.1 \mu \mathrm{m})$ for our initial trials. The choice for the incidence angle was determined by our UHV experimental setup that allows 45 degrees light incidence. The thin film samples were conformally deposited onto standard gratings (Fig. 1). We have deposited $\mathrm{MgO}$ cap layers over the $\mathrm{Ag}$ films, since it was reported to lower the work-function [1].
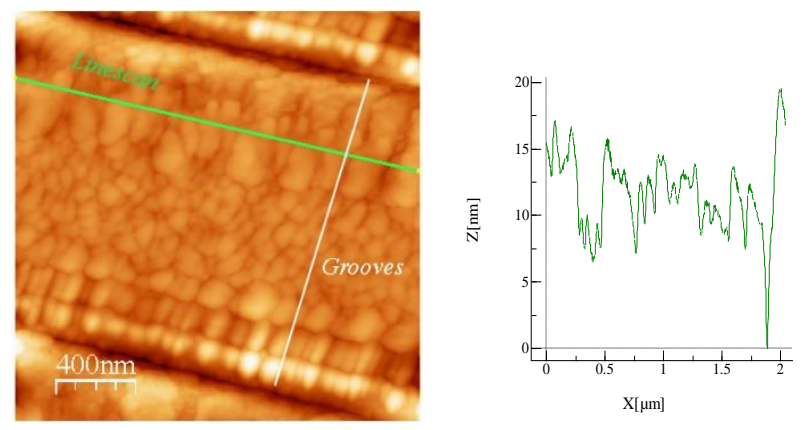

Figure 1. (Left) AFM image of a silver film capped with $\mathrm{MgO}$ deposited on a standard grating with 1.6 microns pitch. We note that the film is conformal with respect to the grating since the grooves are still noticeable; (right) a linescan extracted from the AFM image shows the typical morphology and roughness of the deposited layers.

The films were characterized and surface Plasmon resonance measurements were carried out to establish the optimal characteristics for our setup geometry (Fig. 2)

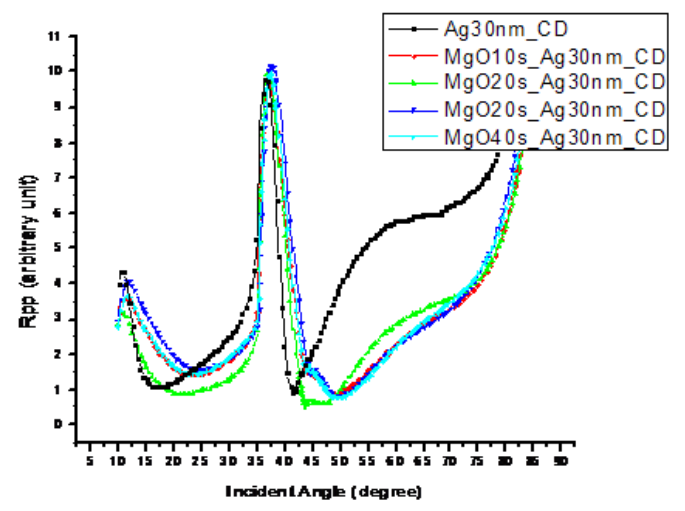


Figure 2. Reflectivity measurements under $\mathrm{cw}$ red light $(\lambda=633 \mathrm{~nm})$, varying the incidence angle for different thin film $\mathrm{Ag}-\mathrm{MgO}$ samples deposited on standard optical gratings with 1.6 $\mu \mathrm{m}$ pitch. We note that the thickness of the $\mathrm{MgO}$ overlayer needs to be fine-tuned to achieve SPPs excitation at 45 degrees.

With optimal deposition conditions established it was found that SPPs excitation was possible illuminating through a glass window, and then similar samples were prepared and transferred in vacuum to the UHV load-lock chamber equipped for photocurrent measurements (Fig. 3).
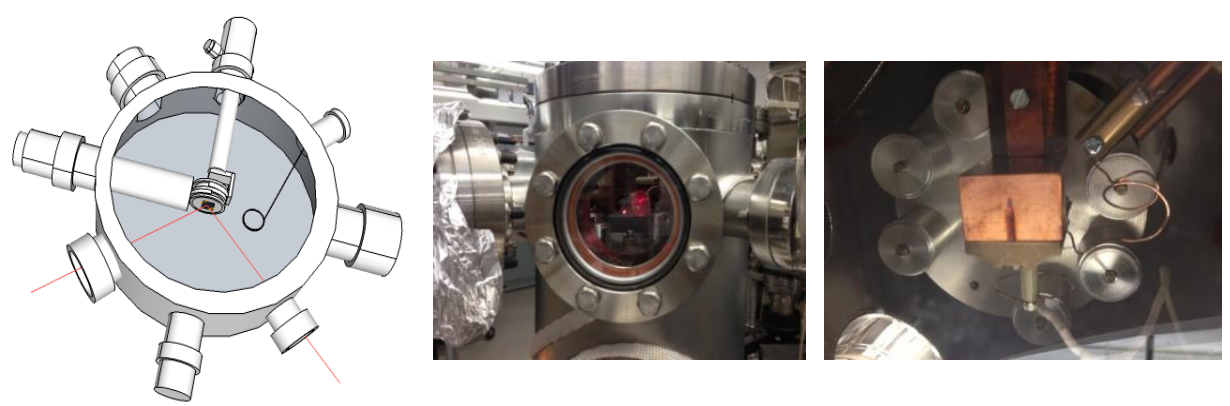

Figure 3. (Left) Sketch of the UHV chamber showing the geometry for illumination and photocurrent measurements; (center) window for incident light; (right) sample mount and Faraday cup detector.

\subsection{Results and conclusions}

Our preliminary results are very encouraging since the photocurrent measured on our plasmonic Ag-MgO samples under low power $(\sim 1 \mathrm{~mW}) \mathrm{cw}$ red light $(633 \mathrm{~nm})$ from a HeNe laser, was $256 \mathrm{pA}$, thus two orders magnitude larger than that reported by A. N. Polyakov et al., i.e. $2.45 \mathrm{pA}$ for a patterned gold sample illuminated with $800 \mathrm{~nm}, 60 \mathrm{fs}, 90 \mathrm{MHz}$ pulsed light at $0.065 \mathrm{GW} / \mathrm{cm} 2$ focused onto a $100 \mu \mathrm{m}$ diameter spot [7].

We are now planning on depositing similar thin film samples tailored for gratings with tighter pitch to enable SPPs excitation at higher light frequencies to further overcome the work function of the metallic layers and hence achieve higher currents. Since significant research has already been carried out exploring multiphoton photoemission from metal cathodes using short laser pulses [7], we are also planning on applying ultrashort laser pulses onto plasmonic samples such as the ones used here to further enhance their QE via n-photon processes. 


\section{References}

[1] T. Koenig, G. H. Simon, H.-P. Rust, and M. Heyde, J. Phys. Chem. C, 113, 11301 (2009)

[2] D. H. Dowell, \&J. F. Schmerge, Phys. Rev. Special Topics - Accelerators and Beams 12 (2009).

[3] T. A Callcott, Phys. Rev. B (1975).

[4] E. Kretschmann \& H. Z. Raether, Naturforsch. A 23, 2135-2136 (1968).

[5] H. Raether, "Surface Plasmons on Smooth and Rough Surfaces and on Gratings," Vol. 111 of Springer Tracts in Modern Physics (Springer-Verlag, Berlin, 1988).

[6] Hari P. Paudel et al., Proc. SPIE 7597, Physics and Simulation of Optoelectronic Devices XVIII, 759706 (2010).

[7] Polyakov A., et al. Phys. Rev. Lett. 110, 076802 (2013) 